\title{
Skeletal muscle metastasis from squamous cell lung cancer was first found by ultrasound: a case report
}

\author{
Lin Du', Ying Sun ${ }^{2}$ \\ ${ }^{1}$ Department of Developmental and Behavioral Pediatrics, ${ }^{2}$ Department of Abdominal Ultrasonography, the First Hospital of Jilin University, \\ Changchun 130021, China \\ Correspondence to: Ying Sun. Department of Ultrasonography, 1st Hospital of Jilin University, No. 71 on Xinmin Street, Chaoyang District, \\ Changchun 130021, China. Email: y_sun@jlu.edu.cn.
}

\begin{abstract}
Skeletal muscle metastasis (SMM) is rare and very difficult to diagnosis using routine ultrasound, computed tomography (CT), and magnetic resonance imaging (MRI), especially in patients without a definitive tumor history. In this report, we describe a 64-year-old man who came to hospital because of pain from a touchable mass at his left thigh. Ultrasonography showed a substantive hypoechoic mass within the left adductor longus muscle with an anechoic area, which was ill-defined and rich in blood supply on color Doppler flow imaging (CDFI). The mass was biopsied under ultrasound guidance. The pathological findings displayed an infiltration of moderately differentiated squamous cell lung cancer (SCLC) and nests of atypical cells. Thoracic plain CT images displayed thickening of the bronchus wall of the right inferior lobe, with obstructive pneumonia and atelectasis at the distal bronchus. The final main diagnosis for this patient was central bronchogenic carcinoma, with intramuscular metastasis of the left adductor longus muscle, and the TNM classification was T2N0M1. Although the incidence of IM originating from SCLC is very rare, patients with an occasional found mass in soft tissue should be examined to exclude SMM, and ultrasoundguided percutaneous biopsy can provide vital information regarding indeterminate soft tissue masses.
\end{abstract}

Keywords: Skeletal muscle; lung cancer; ultrasound; case report

Submitted Aug 20, 2019. Accepted for publication Dec 16, 2019.

doi: $10.21037 /$ tcr.2019.12.55

View this article at: http://dx.doi.org/10.21037/tcr.2019.12.55

\section{Introduction}

Skeletal muscle metastasis (SMM) is rare and very difficult to diagnosis using routine ultrasound, computed tomography (CT), and or magnetic resonance imaging (MRI), especially in patients without a definitive tumor history (1). Intramuscular metastases (IM) from squamous cell lung cancer (SCLC) are extremely rare, although the incidence of lung cancer is very high all over the world, with lung cancer causing the most tumor-related deaths among both males and females (2). The most frequent metastatic sites of lung cancer are the nervous system, bone, liver, respiratory system, and adrenal gland (3). There are only a few of case reports concerning IM from SCLC (4). In this report, we describe a patient with SMM as the initial manifestation of lung cancer.

\section{Case presentation}

A 64-year-old man was referred to the Department of Articular Surgery because of pain from a touchable mass at his left thigh. His past medical history was uneventful, except for a history of smoking for 45 years. He denied any family history with particular disease. None of systematic physical examination was done before. This visit, physical examination revealed a palpable mass with haphalgesia in the medial left thigh that was solid and hard. There was no palpated superficial lymphadenopathy and no auscultatory abnormality in either lung. Ultrasonography showed a substantive hypoechoic mass within the left adductor longus muscle with an anechoic area, which was ill-defined and rich in blood supply on CDFI (Figure 1). On MRI, the mass showed a mild hyper-intense signal on T2-weighted 


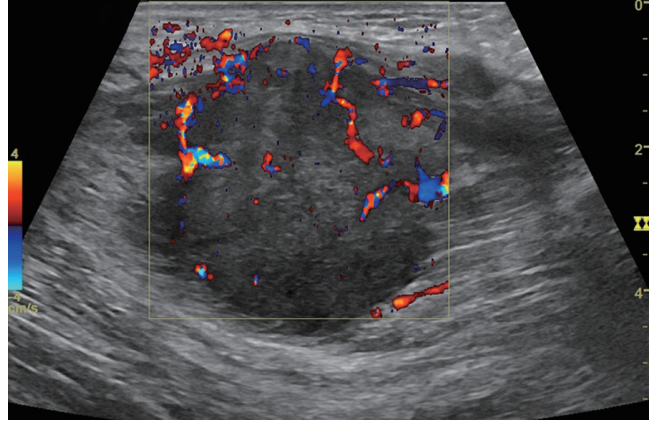

Figure 1 Ultrasonography showed a substantive hypoechoic mass within the left adductor longus muscle with an anechoic area, which was ill-defined and rich in blood supply on color Doppler flow imaging.

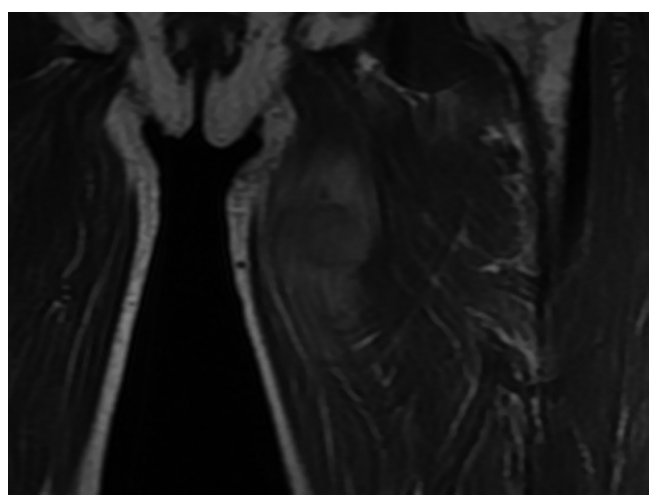

Figure 2 MRI. The mass showed a mild hyper-intense signal on T2-weighted imaging (T2WI) and mild low intensity on T1weighted imaging (T1WI), with a diameter of approximately $4.0 \mathrm{~cm}$. MRI, magnetic resonance imaging.

imaging (T2WI), and mild low intensity on T1-weighted imaging (T1WI) (Figure 2). The mass was biopsied under ultrasound guidance. The pathological findings displayed an infiltration of moderately differentiated SCLC and nests of atypical cells (Figure 3). The immunochemistry results were as follows: CK5/6 (+), CK7 (+), P63 (+), and TTF-1 (-) (Figure 4).

Considering the patient's long history of smoking, a thoracic plain CT examination was performed. CT images displayed thickening of the bronchus wall of the right inferior lobe, with obstructive pneumonia and atelectasis at the distal bronchus (Figure 5). There was no mediastinal and hilar lymphadenectasis. Therefore, the CT findings were consistent with the diagnosis of central bronchogenic carcinoma. In order to eliminate the

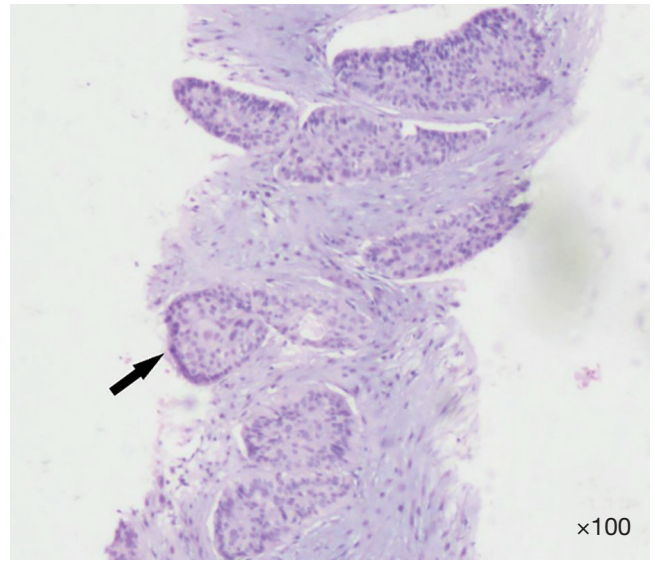

Figure 3 Core biopsy histology (hematoxylin-eosin staining, $\times 100$ ) showing medium differentiated squamous cell carcinoma with infiltration and nests of atypical cells indicated by arrow. Though there were no keratin pearls found in the specimen, immunohistochemistry staining confirmed their presence and excluded other possibilities.

possibility of remote organ metastasis, craniocerebral CT and abdominal CT, along with lymph node scanning by ultrasound and emission computed tomography (ECT), were performed. No suspicious positive findings were observed. The laboratory test results for tumor markers were as follows: squamous cell carcinoma antigen (SCC-ag) $7.96 \mathrm{ng} / \mathrm{mL}$, carcinoembryonic antigen (CEA) $55.41 \mathrm{ng} / \mathrm{mL}$, CYFRA21-1 $6.64 \mathrm{ng} / \mathrm{mL}$, and cancer antigen (CA125) $37.86 \mathrm{U} / \mathrm{mL}$. Therefore, the final main diagnosis for this patient was central bronchogenic carcinoma, with intramuscular metastasis of the left adductor longus muscle, and the TNM classification was T2N0M1. Because of the financial situation of his family, he rejected bronchoscopy, $\mathrm{PET} / \mathrm{CT}$ examination, and resection of the original lung tumor and only received chemotherapy with a gemcitabinecisplatin combination in his local hospital, afterwards loss to follow-up.

The participant gave his informed written consent before taking part in the study. The research protocol observed the tenets of the Declaration of Helsinki and was approved by the ethics committee of the First Hospital of Jilin University (No. 2017-428).

\section{Discussion}

Skeletal muscle is a rare site for hematogenous metastases from epithelial neoplasms (1), and solitary IM in patients 

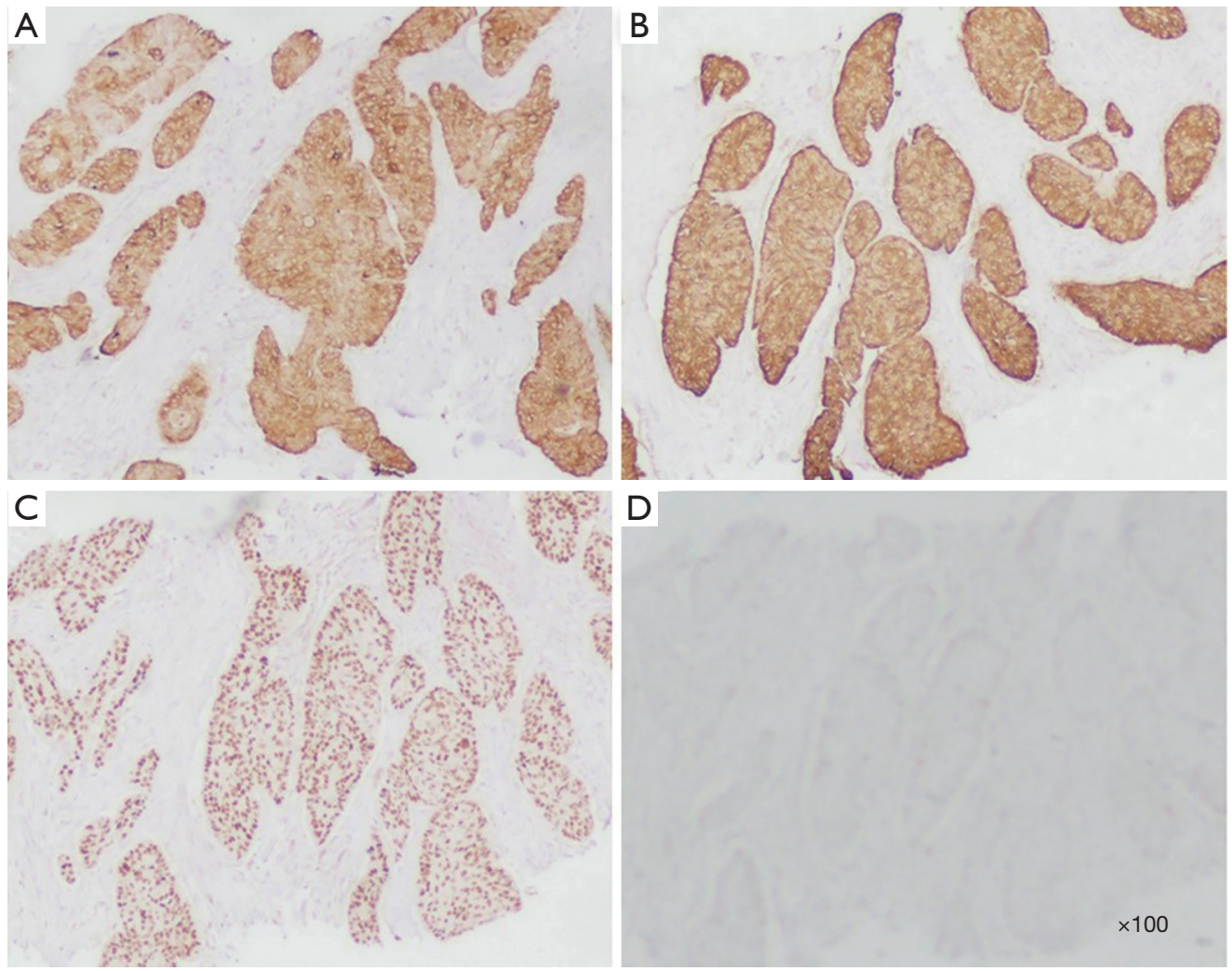

Figure 4 (A) CK56 (+); (B) CK7 (+); (C) P63 (+); and (D) TFF-1 (-). Positive staining for CK7 and CK56 indicated squamous cell carcinoma, while negative staining for TFF-1 negative excluded adenocarcinoma. CK7 positivity is not normal in squamous cell carcinoma and has an intersection with transitional cell carcinoma. The patient's urinary system had no abnormal findings; therefore, transitional cell carcinoma was excluded. Magnification $\times 100$.

with lung cancer is even rarer (5). About $40-50 \%$ of primary lesions for IM are lung cancer (1). The most common histological type is adenocarcinoma of the lung and gastrointestinal tract (35\%) (1). Arpaci et al. reported an SMM incidence of $0.16 \%$ among 2,557 patients with lung cancer (1). The most frequently involved muscles are the thigh, iliopsoas, and paraspinous muscles (5).

Metastasis to the skeletal muscle may be more prevalent than previously estimated. It may be an incidental finding on ultrasound or CT. Ultrasound is a noninvasive, economical, and repeatable method for identifying soft tissue masses; therefore, it is usually adopted as the first line examination in clinic (6). Ultrasound-guided percutaneous biopsy is very valuable for finding indeterminate masses, which can provide a credible pathological diagnosis and powerful evidence for tumor staging and prognostic evaluating (6). The most common appearance on contrastenhanced CT is a mass with rim enhancement and central hypoattenuation (1). Any intramuscular mass appearing in an oncology patient that shows extensive tumor enhancement, central necrosis, and peritumoral edema on MRI is highly suspicious for SMM (1). Metastasis to the skeletal muscle is one of the deteriorating signs for the prognosis of oncology patients. Mathis et al. reported that most cases of IM are observed in males and usually occur during disease progression for oncology patients (5). So et al. estimated that SMM represents approximately $0.41 \%$ of all metastases originating from lung cancer and detected on PET/CT (7).

Neoplasia is quite rare in myology. It is thought that muscle movement, $\mathrm{pH}$, and the muscle's capability to remove the lactic acid produced by a tumor contribute to the resistance of skeletal muscles to the metastatic process (8). According to the "seed and soil" hypothesis, lung cancer cells are the "seed", while the muscle is the "soil" (8). Malignant cells originating from lung cancer express high 


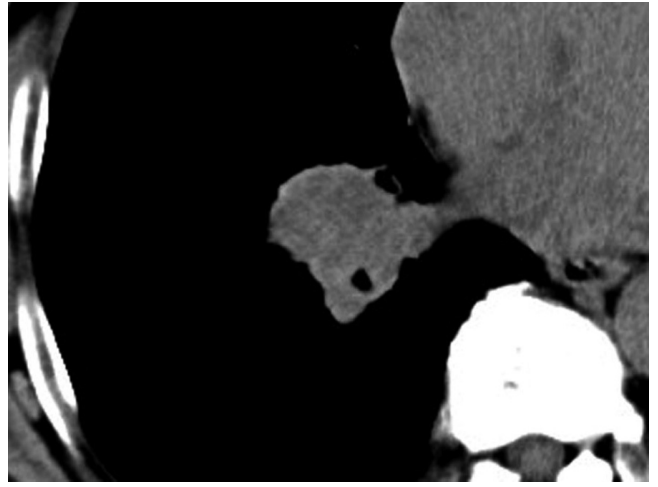

Figure 5 The wall of the bronchus to the right inferior lobe showed thickening, and the diameter of the bronchus got was obstructed by pneumonia.

levels of Angpt14 and VEGF-A factors, which hinder the aggregation of vascular endothelial cells and facilitates extravasation; this may be one reason why the seed can leave the original soil (9). The seeds travel far to muscle, with a high risk to the optimal soil. Our patient had no lymph metastasis, and thus, we suppose that the metastatic route was via the blood vessels. Regardless, the occurrence of SMM from lung cancer suggests a bad prognosis (10). Pop et al. (10) reported that the survival rate is influenced by the number of SMM cases. The formerly reported patients usually had large original tumors and mediastinal lymph node metastasis, or SMM occurring an amount of time after the resection of the original tumor $(11,12)$. However, our patient had non-respiratory symptoms, and the palpable painful mass in the left thigh was the only complaint. The diameter of the original lung tumor in our patient was only $2 \mathrm{~cm}$, without any mediastinal lymph node metastasis. ${ }^{18}$ FDG PET/CT has excellent diagnostic value for distant metastases in patients with lung cancer (13). Unfortunately, our patient rejected PET/CT examination and resection of the original lung tumor and received chemotherapy with the gemcitabine-cisplatin combination, owing to the financial situation of his family. Even though there exists a case report of successful resection of stage IV NSCLC with muscle metastasis (12), the optimal mode of treatment and prognosis is undefined for these patients because of its rare incidence. The limitation of this case report is that follow up of the patient was incomplete due to the hopelessness to the prognosis of disease and refuse to receive any social help.

\section{Conclusions}

Although the incidence of IM originating from SCLC is very rare, patients with an occasional found mass in soft tissue should be examined to exclude SMM, and ultrasoundguided percutaneous biopsy can provide vital information regarding indeterminate soft tissue masses.

\section{Acknowledgments}

Funding: This work was supported by the Science and Technology Innovation Plan of Jilin Provincial Science \& Technology Department, China. No. 20170204047SF.

\section{Footnote}

Conflicts of Interest: All authors have completed the ICMJE uniform disclosure form (available at http://dx.doi. org/10.21037/tcr.2019.12.55). The authors have no conflicts of interest to declare.

Ethical Statement: The authors are accountable for all aspects of the work in ensuring that questions related to the accuracy or integrity of any part of the work are appropriately investigated and resolved. All procedures performed in studies involving human participants were in accordance with the ethical standards of the institutional and/or national research committee(s) and with the Helsinki Declaration (as revised in 2013). Written informed consent was obtained from the patient for publication of this manuscript and any accompanying images.

Open Access Statement: This is an Open Access article distributed in accordance with the Creative Commons Attribution-NonCommercial-NoDerivs 4.0 International License (CC BY-NC-ND 4.0), which permits the noncommercial replication and distribution of the article with the strict proviso that no changes or edits are made and the original work is properly cited (including links to both the formal publication through the relevant DOI and the license). See: https://creativecommons.org/licenses/by-nc-nd/4.0/.

\section{References}

1. Arpaci T, Ugurluer G, Akbas T, et al. Imaging of the skeletal muscle metastases. Eur Rev Med Pharmacol Sci 2012;16:2057-63. 
2. Siegel RL, Miller KD, Jemal A. Cancer statistics, 2015. CA Cancer J Clin 2015;65:5-29.

3. Riihimäki $M$, Hemminki A, Fallah $M$, et al. Metastatic sites and survival in lung cancer. Lung Cancer 2014;86:78-84.

4. Song SH, Oh YJ, Kim YN, et al. Squamous cell carcinoma of the lung with simultaneous metastases to peritoneum and skeletal muscle. Thorac Cancer 2014;5:101-3.

5. Mathis S, Fromont-Hankard G, du Boisguéheneuc F, et al. Muscular metastasis. Rev Neurol (Paris) 2010;166:295-304.

6. Kim SY, Chung HW, Oh TS, et al. Practical Guidelines for Ultrasound-Guided Core Needle Biopsy of Soft-Tissue Lesions: Transformation from Beginner to Specialist. Korean J Radiol 2017;18:361-9.

7. So Y, Yi JG, Song I, et al. Detection of skeletal muscle metastasis: torso FDG PET-CT versus contrast-enhanced chest or abdomen CT. Acta Radiol 2015;56:860-6.

8. de Groot AE, Roy S, Brown JS, et al. Revisiting Seed

Cite this article as: Du L, Sun Y. Skeletal muscle metastasis from squamous cell lung cancer was first found by ultrasound: a case report. Transl Cancer Res 2019;8(8):2936-2940. doi: $10.21037 /$ tcr.2019.12.55 and Soil: Examining the Primary Tumor and Cancer Cell Foraging in Metastasis. Mol Cancer Res 2017;15:361-70.

9. Padua $\mathrm{D}$, Zhang XH, Wang Q, et al. TGFbeta primes breast tumors for lung metastasis seeding through angiopoietin-like 4. Cell 2008;133:66-77.

10. Pop D, Nadeemy AS, Venissac N, et al. Skeletal muscle metastasis from non-small cell lung cancer. J Thorac Oncol 2009;4:1236-41.

11. Kwas HH, Zendah I, Ghedira H. Skeletal muscle metastases from lung cancer. Asian Cardiovasc Thorac Ann 2013;21:741-3.

12. Mogi A, Kosaka T, Yamaki E, et al. Successful resection of stage IV non-small cell lung cancer with muscle metastasis as the initial manifestation: a case report. Ann Thorac Cardiovasc Surg 2012;18:468-71.

13. Li J, Xu W, Kong F, et al. Meta-analysis: accuracy of 18FDG PET-CT for distant metastasis staging in lung cancer patients. Surg Oncol 2013;22:151-5. 